\title{
Controllability of Second-Order Semilinear Impulsive Stochastic Neutral Functional Evolution Equations
}

\author{
Lei Zhang, ${ }^{1,2}$ Yongsheng Ding, ${ }^{1,2}$ Tong Wang, ${ }^{1,2}$ \\ Liangjian $\mathrm{Hu}^{3}$ and Kuangrong $\mathrm{Hao}^{1,2}$ \\ ${ }^{1}$ College of Information Sciences and Technology, Donghua University, Shanghai 201620, China \\ ${ }^{2}$ Engineering Research Center of Digitized Textile E Fashion Technology, Ministry of Education, \\ Donghua University, Shanghai 201620, China \\ ${ }^{3}$ Department of Mathematics, Donghua University, Shanghai 201620, China
}

Correspondence should be addressed to Yongsheng Ding, ysding@dhu.edu.cn

Received 17 June 2012; Accepted 7 August 2012

Academic Editor: Bo Shen

Copyright (C) 2012 Lei Zhang et al. This is an open access article distributed under the Creative Commons Attribution License, which permits unrestricted use, distribution, and reproduction in any medium, provided the original work is properly cited.

\begin{abstract}
We consider a class of impulsive neutral second-order stochastic functional evolution equations. The Sadovskii fixed point theorem and the theory of strongly continuous cosine families of operators are used to investigate the sufficient conditions for the controllability of the system considered. An example is provided to illustrate our results.
\end{abstract}

\section{Introduction}

Controllability, as a fundamental concept of control theory, plays an important role both in stochastic and deterministic control problems. The study of controllability of linear and nonlinear systems represented by infinite-dimensional systems in Banach spaces has been raised by many authors recently, see Chang [1], Sakthivel [2], Ren and Sakthivel [3], Ntouyas and Regan [4], Kang et al. [5], Sakthivel and Mahmudov [6], and Shubov et al. [7]. With the help of fixed point theorem, Luo [8,9] and Burton [10-13] have investigated the problem of controllability of the systems in Banach spaces.

Recently, stochastic partial differential equations (SPDEs) arise in the mathematical modeling of various fields in physics and engineering science cited by Sobczyk [14]. Among them, several properties of SPDEs such as existence, controllability, and stability are studied for the first-order equations. But in many situations, it is useful to investigate the secondorder abstract differential equations directly rather than to convert them to first-order systems introduced by Fitzgibbon [15]. The second-order stochastic differential equations are the right 
model in continuous time to account for integrated processes that can be made stationary. For instance, it is useful for engineers to model mechanical vibrations or charge on a capacitor or condenser subjected to white noise excitation by second-order stochastic differential equations. A useful tool for the study of abstract second-order equations is the fixed point theory and the theory of strongly continuous cosine families.

In the past decades, the theory of impulsive differential equations or inclusions is emerging as an active area of investigation due to the application in area such as mechanics, electrical engineering, medicine biology, and ecology, see Benchohra and Henderson [16], Liu and Willms [17], Hernández et al. [18], Prato and Zabczyk [19], and Fattorini [20]. As an adequate model, impulsive differential equations are used to study the evolution of processes that are subject to sudden changes in their states.

The focus of this paper is the controllability of mild solutions for a class of impulsive neutral second-order stochastic evolution equations of the form:

$$
\begin{gathered}
d\left[x^{\prime}(t)-D\left(x_{t}\right)\right]=\left[A x(t)+B u(t)+f\left(t, x_{t}\right)\right] d t+g\left(t, x_{t}\right) d w(t), \quad t \in[0, T], \quad t \neq t_{k} \\
\Delta x\left(t_{k}\right)=I_{k}\left(x\left(t_{k}\right)\right), \quad \Delta x^{\prime}\left(t_{k}\right)=\tilde{I}_{k}\left(x\left(t_{k}\right)\right), \quad k=1, \ldots, n, x(0)=\phi, x^{\prime}(0)=y_{0} .
\end{gathered}
$$

Here, $x(\cdot)$ is a stochastic process taking values in a real separable Hilbert space $H$ with inner product $(\cdot, \cdot)$ and norm $\|\cdot\| . A: D(A) \subset H \rightarrow H$ is the infinitesimal generator of a strongly continuous cosine family on $H$. W is a given $K$-valued Wiener process with a finite trace nuclear covariance operator $Q \geq 0$ defined on a filtered complete probability space $\left(\Omega, F,\left\{F_{t}\right\}_{t \geq 0}, P\right)$ and $K$ is another separable Hilbert space with inner product $(\cdot, \cdot)_{K}$ and norm $\|\cdot\|_{K}$. The fixed time $t_{k}, k=1, \ldots, n$, satisfies $0<t_{1}<\cdots<t_{n}<T, x\left(t_{k}^{+}\right)$and $x\left(t_{k}^{-}\right)$denote the right and left limits of $x(t)$ at $t=t_{k}$, and $\Delta x\left(t_{k}\right)=x\left(t_{k}^{+}\right)-x\left(t_{k}^{-}\right)$represents the jump in the state $x$ at time $t_{k}$, where $I_{k} \in C(H, H)(k=1,1,2, \ldots, m)$ are bounded which determine the size of the jump. Similarly $x^{\prime}\left(t_{k}^{+}\right)$and $x^{\prime}\left(t_{k}^{-}\right)$denote, respectively, the right and left limits of $x^{\prime}$ at $t_{k} . f, B, g$ are appropriate mappings specified later; $x_{0}$ and $y_{0}$ are $F_{0}$-measurable random variables with finite second moment. The main contributions are as follows. The Sadovskii fixed point theorem and the theory of strongly continuous cosine families of operators are used to investigate the sufficient conditions for the controllability of the system considered. The differences of using the fixed point theorem between our proposed method and others are that Sadovskii fixed point theorem is much easier in application, and the condition is easier to be satisfied than other fixed point theorem. To our best knowledge, there are few works about the controllability for mild solutions to second-order semilinear impulsive stochastic neutral functional evolution equations, motivated by the previous problems, our current consideration is on second-order semilinear impulsive stochastic neutral functional evolution equations. We will apply the Sadovskii fixed point theorem to investigate the controllability of mild solution of this class of equations.

The rest of this paper is arranged as follows. In Section 2, we briefly present some basic notations and preliminaries. Section 3 is devoted to the controllability of mild solutions for the system (1.1) and an example is given to illustrate our results in Section 4. Conclusion is given in Section 5.

\section{Preliminaries}

In this section, we briefly recall some basic definitions and results for stochastic equations in infinite dimensions and cosine families of operators. We refer to Prato and Zabczyk [19] 
and Fattorini [20] for more details. Throughout this paper, let $L(K, H)$ be the set of all linear bounded operators from $K$ into $H$, equipped with the usual operator norm $\|\cdot\|$. Let $(\Omega, F, P)$ be a complete probability space furnished with a normal filtration $\left\{F_{t}\right\}_{t \geq 0}$. Suppose $\left\{\beta_{k}\right\}_{k \geq 1}$ is a sequence of real independent one-dimensional standard Brownian motions over $(\Omega, F, P)$. Set

$$
W(t)=\sum_{k=1}^{\infty} \sqrt{\lambda_{k}} \beta_{k}(t) e_{k}, \quad t \geq 0
$$

where $\left\{e_{k}\right\}_{k \geq 1}$ is the complete orthonormal system in $K$ and $\lambda_{k}, k \geq 1$, a bounded sequence of nonnegative real numbers. Let $Q \in L(K, K)$ be an operator defined by $Q e_{k}=\lambda_{k} e_{k}, k=1,2, \ldots$, with $\operatorname{tr} Q=\sum_{k=1}^{\infty} \lambda_{k}<\infty$. The $K$-valued stochastic process $W=\left(W_{t}\right)_{t \geq 0}$ is called a $Q$-Wiener process. Let $L_{2}^{0}=L_{2}\left(Q^{1 / 2} K, H\right)$ be the space of all Hilbert-Schmidt operators from $Q^{1 / 2} K$ to $H$ with the inner product $\langle\varphi, \phi\rangle_{L_{2}^{0}}=\operatorname{tr}\left[\varphi Q \phi^{*}\right]$.

The collection of all strongly measurable, square-integrable $H$-valued random variables, denoted by $L^{2}(\Omega, H)$, is a Banach space equipped with norm $\|x\|_{L^{2}}=\left(E\|x\|^{2}\right)^{1 / 2}$. An important subspace of $L^{2}(\Omega, H)$ is given by

$$
L_{0}^{2}(\Omega, H)=\left\{L^{2}(\Omega, H) \ni x \text { is } F_{0}-\text { measurable }\right\} .
$$

Let

$$
\begin{aligned}
\wp & :=D([0, T], H) \\
& =\left\{x:[0, T] \longrightarrow H,\left.x\right|_{\left(t_{k}, t_{k+1}\right]} \in C\left(\left(t_{k}, t_{k+1}\right], H\right), \text { and there exists } x\left(t_{k}^{+}\right) \text {for } k=1,2, \ldots, n\right\}, \\
\bar{\wp} & :=\bar{D}([0, T], H) \\
& =\left\{x \in \wp,\left.x\right|_{\left(_{k}, t_{k+1}\right]} \in C^{1}\left(\left(t_{k}, t_{k+1}\right], H\right), \text { and there exists } x^{\prime}\left(t_{k}^{+}\right) \text {for } k=1,2, \ldots, n\right\} .
\end{aligned}
$$

It is obvious that $D([0, T], H)$ and $\bar{D}([0, T], H)$ are Banach spaces endowed with the norm

$$
\|x\|_{\mathscr{\wp}}=\left(\sup _{t \in[0, T]} E\|x(t)\|^{2}\right)^{1 / 2}
$$

and $\|x\|_{\bar{\wp}}=\|x\|_{\mathscr{\wp}}+\left\|x^{\prime}\right\|_{\wp^{\prime}}$, respectively.

To simplify the notations, we put $t_{0}=0, t_{m+1}=T$, and for $u=H_{2}$, we denote by $\tilde{u}_{k} \in C\left(\left[t_{k}, t_{k+1}\right], L^{2}(\Omega, H)\right), k=0,1, \ldots, m$, the function given by

$$
\tilde{u}_{k}(t)= \begin{cases}u(t), & t \in\left(t_{k}, t_{k+1}\right], \\ u\left(t_{k}^{+}\right), & t=t_{k} .\end{cases}
$$

Moreover, for $B \subset H_{2}$ we denote $\widetilde{B}_{k}=\left\{\widetilde{u}_{k}: u \in B\right\}, k=1, \ldots, m$. To prove our results, we need the following lemma introduced in Hernández et al. [18]. 
Lemma 2.1. $A$ set $B \subset \wp$ is relatively compact in $\wp$ if and only if the set $\widetilde{B}_{k}$ is relatively compact in $C\left(\left[t_{k}, t_{k+1}\right], H\right)$, for every $k=0,1, \ldots, m$.

Now, we recall some facts about cosine families of operators, see Fattorini [20] and Travis and Webb [21].

Definition 2.2. (1) The one-parameter family $\{C(t): t \in \mathbb{R}\} \subset L(H, H)$ is said to be a strongly continuous cosine family if the following hold:

(1) $C(0)=I$;

(2) $C(t) x$ is continuous in $t$ on $\mathbb{R}$ for any $x \in H$;

(3) $C(t+s)+C(t-s)=2 C(t) C(s)$ for all $t, s \in \mathbb{R}$. defined by

(2) The corresponding strongly continuous sine family $\{S(t): t \in \mathbb{R}\} \subset L(H, H)$ is

$$
S(t) x=\int_{0}^{t} C(s) x d s, \quad t \in \mathbb{R}, x \in H .
$$

(3) The (infinitesimal) generator $A: H \rightarrow H$ of $\{C(t): t \in R\}$ is given by

$$
A x=\left.\frac{d^{2}}{d t^{2}} C(t) x\right|_{t=0}
$$

for all $x \in D(A)=\left\{x \in H: C(\cdot) x \in C^{2}(\mathbb{R}, H)\right\}$.

It is known that the infinitesimal generator $A$ is a closed, densely defined operator on $H$, and the following properties hold, see Travis and Webb [21].

Proposition 2.3. Suppose that $A$ is the infinitesimal generator of a cosine family of operators $\{C(t)$ : $t \in \mathbb{R}\}$. Then, the following hold

(i) There exist a pair of constants $M_{A} \geq 1$ and $\alpha \geq 0$ such that $\|C(t)\| \leq M_{A} e^{\alpha|t|}$ and hence, $\|S(t)\| \leq M_{A} e^{\alpha|t|}$.

(ii) $A \int_{s}^{r} S(u) x d u=[C(r)-C(s)] x$, for all $0 \leq s \leq r<\infty$.

(iii) There exist $N \geq 1$ such that $\|S(s)-S(r)\| \leq N\left|\int_{s}^{r} e^{\alpha|s|} d s\right|$, for all $0 \leq s \leq r<\infty$.

The uniform boundedness principle: as a direct consequence we see that both $\{C(t): t \in[0, T]\}$ and $\{S(t): t \in[0, T]\}$ are uniformly bounded by $M^{*}=M_{A} e^{\alpha|T|}$.

At the end of this section we recall the fixed point theorem of Sadovskii [22] which is used to estimate the controllability of the mild solution to the system (1.1).

Lemma 2.4. Let $\Phi$ be a condensing operator on a Banach space $H$. If $\Phi(N) \subset N$ for a convex, closed, and bounded set $N$ of $H$, then $\Phi$ has a fixed point in $H$. 


\section{Main Results}

In this section we consider the system (1.1). We first present the definition of mild solutions for the system.

Definition 3.1. An $F_{t}$-adapted stochastic process $x(t):[0, T] \rightarrow H$ is said to be a mild solution of the system (1.1) if

(1) $x_{0}, y_{0} \in L_{0}^{2}(\Omega, H)$;

(2) $\Delta x\left(t_{k}\right)=x\left(t_{k}^{+}\right)-x\left(t_{k}^{-}\right)=I_{k}\left(x\left(t_{k}\right)\right), \Delta x^{\prime}\left(t_{k}\right)=x^{\prime}\left(t_{k}^{+}\right)-x^{\prime}\left(t_{k}^{-}\right)=\tilde{I}_{k}\left(x\left(t_{k}\right)\right), k=1, \ldots, n$;

(3) $x(t)$ satisfies the following integral equation:

$$
\begin{aligned}
x(t)= & C(t) \phi(0)+S(t)\left[y_{0}-D(0, \phi)\right]+\int_{0}^{t} C(t-s) D\left(s, x_{s}\right) d s \\
& +\int_{0}^{t} S(t-s) B u(s) d s+\int_{0}^{t} S(t-s) f\left(s, x_{s}\right) d s \\
& +\int_{0}^{t} S(t-s) g\left(s, x_{s}\right) d W(s)+\sum_{0<t_{k}<t} C\left(t-t_{k}\right) I_{k}\left(x\left(t_{k}\right)\right) \\
& +\sum_{0<t_{k}<t} S\left(t-t_{k}\right) \tilde{I}_{k}\left(x\left(t_{k}\right)\right) .
\end{aligned}
$$

In this paper, we will work under the following assumptions.

(A1) The cosine family of operators $\{C(t): t \in[0, T]\}$ on $H$ and the corresponding sine family $\{S(t): t \in[0, T]\}$ are compact for $t>0$, and there exists a positive constant $M$ such that

$$
\|C(t)\| \leq M, \quad\|S(t)\| \leq M
$$

(A2) $D, f, g$ are continuous functions, and there exist some positive constants $M_{D}, M_{f}, M_{g}$, such that $D, f, g$ satisfy the following Lipschitz condition:

$$
\begin{gathered}
\|D(t, \varphi)-D(t, \phi)\| \leq M_{D}\|\varphi-\phi\|, \\
\|f(t, \varphi)-f(t, \phi)\| \leq M_{f}\|\varphi-\phi\|, \\
\|g(t, \varphi)-g(t, \phi)\| \leq M_{g}\|\varphi-\phi\|,
\end{gathered}
$$


for all $\varphi, \phi \in H, k=1, \ldots, n$ and $t \in[0, T]$, and there exist positive constants $\bar{M}_{D}, \bar{M}_{f}, \bar{M}_{g}$ that satisfy the following linear growth condition:

$$
\begin{gathered}
\|D(t, \varphi)\|^{2} \leq \bar{M}_{D}\left(\|\varphi\|^{2}+1\right), \\
\|f(t, \varphi)\|^{2} \leq \bar{M}_{f}\left(\|\varphi\|^{2}+1\right), \\
\|g(t, \varphi)\|^{2} \leq \bar{M}_{g}\left(\|\varphi\|^{2}+1\right)
\end{gathered}
$$

for all $\varphi, \phi \in H, k=1, \ldots, n$ and $t \in[0, T]$.

(A3) $I_{k}, \widetilde{I}_{k}: H \rightarrow H$ are continuous and there exist positive constants $M_{k}, N_{k}$ such that

$$
\left\|I_{k}(x)-I_{k}(y)\right\| \leq M_{k}\|x-y\|^{2}, \quad\left\|\tilde{I}_{k}(x)-\tilde{I}_{k}(y)\right\| \leq N_{k}\|x-y\|^{2}
$$

for each $x, y \in H, k=1, \ldots, n$.

(A4) $B$ is a continuous operator from $\Omega$ to $H$ and the linear operator $W: L_{0}^{2}(\Omega, H) \rightarrow X$ defined by

$$
W u=\int_{0}^{T} S(T-s) B u(s) d s
$$

has a bounded invertible operator $W^{-1}$ which takes values in $L_{0}^{2}(\Omega, H) /$ ker $W$ such that $\|B\| \leq M_{1},\left\|W^{-1}\right\| \leq M_{2}$, for some positive constants $M_{1}, M_{2}$.

We formulate and prove conditions for the approximate controllability of semilinear control differential systems

Theorem 3.2. Assume that (A1)-(A4) are satisfied and $x_{0}, y_{0} \in L_{0}^{2}(\Omega, H)$, then the system (1.1) is controllable on $[0, T]$ provided that

$$
\begin{aligned}
& 8 M^{2}\left[T \bar{M}_{D}^{2}+T \bar{M}_{f}^{2}+\operatorname{tr}(Q) \bar{M}_{g}^{2}+2 M^{2} \sum_{k=1}^{n} M_{k}+2 M^{2} \sum_{k=1}^{n} N_{k}\right. \\
& \left.\quad+8 M_{2}\left(T \bar{M}_{D}^{2}+T \bar{M}_{f}^{2}+\operatorname{tr}(Q) \bar{M}_{g}^{2}+2 M^{2} \sum_{k=1}^{n} M_{k}+2 M^{2} \sum_{k=1}^{n} N_{k}\right)\right]<1 .
\end{aligned}
$$


Proof. Define the control process with final value $\xi=x(T)$

$$
\begin{aligned}
u_{x}^{T}(t)=W^{-1}\left\{\xi-S(T)\left[y_{0}-D(0, \phi)\right]-C(T) \phi(0)-\int_{0}^{T} C(T-s) D\left(s, x_{s}\right) d s\right. \\
-\int_{0}^{T} S(T-s) f\left(s, x_{s}\right) d s-\int_{0}^{T} S(T-s) g\left(s, x_{s}\right) d W(s) \\
\left.-\sum_{0<t_{k}<t} C\left(T-t_{k}\right) I_{k}\left(x\left(t_{k}\right)\right)-\sum_{0<t_{k}<t} S\left(T-t_{k}\right) \tilde{I}_{k}\left(x\left(t_{k}\right)\right)\right\}(t) .
\end{aligned}
$$

Let $B_{N}=\left\{x \in H_{2}:\|x\|_{\mathscr{\delta}}^{2} \leq N\right\}$, for every positive integer $N$. It is clear that $B_{N}$ is a bounded closed convex set in $\mathrm{H}_{2}$ for each $N$. Define an operator $\pi: \mathrm{H}_{2} \rightarrow \mathrm{H}_{2}$ by

$$
\begin{aligned}
(\pi x)(t)= & C(t) \phi(0)+S(t)\left[y_{0}-D(0, \phi)\right]+\int_{0}^{t} C(t-s) D\left(s, x_{s}\right) d s+\int_{0}^{t} S(t-s) B u(s) d s \\
& +\int_{0}^{t} S(t-s) f\left(s, x_{s}\right) d s+\int_{0}^{t} S(t-s) g\left(s, x_{s}\right) d W(s) \\
& +\sum_{0<t_{k}<t} C\left(t-t_{k}\right) I_{k}\left(x\left(t_{k}\right)\right)+\sum_{0<t_{k}<t} S\left(t-t_{k}\right) \tilde{I}_{k}\left(x\left(t_{k}\right)\right) .
\end{aligned}
$$

Now let us show that $\pi$ has a fixed point in $H_{2}$ which is a solution of (1.1) by Lemma 2.4. This will be done in the next lemmas.

Lemma 3.3. There exists a positive integer $N$ such that $\pi\left(B_{N}\right) \subset B_{N}$.

Proof. This proof can be done by contradiction. In fact, if it is not true, then for each positive number $N$ and $t^{N} \in[0, T]$, there exists a function $x^{N} \in B_{N}$, but $\pi\left(x^{N}\right)\left(t^{N}\right) \notin B_{N}$. That is, $E\left\|\pi\left(x^{N}\right)\left(t^{N}\right)\right\|^{2}>N$. By applying assumptions (A1)-(A4) one can obtain the following estimates:

$$
\begin{aligned}
E\left\|\sum_{0<t_{k}<t^{N}} S\left(t^{N}-t_{k}\right) \tilde{I}_{k}\left(x^{N}\left(t_{k}\right)\right)\right\|^{2} & \leq N M^{2} \sum_{0<t_{k}<T} E\left\|\tilde{I}_{k}\left(x^{N}\left(t_{k}\right)\right)-\tilde{I}_{k}(0)+\tilde{I}_{k}(0)\right\| \\
& \leq 2 N M^{2}\left(\sum_{k=1}^{N} N_{k} E\left\|x^{N}\left(t_{k}\right)\right\|^{2}+\sum_{k=1}^{N}\left\|\tilde{I}_{k}(0)\right\|^{2}\right), \\
E\left\|\sum_{0<t_{k}<t^{N}} C\left(t^{N}-t_{k}\right) I_{k}\left(x^{N}\left(t_{k}\right)\right)\right\|^{2} & \leq N M^{2} \sum_{0<t_{k}<T} E\left\|I_{k}\left(x^{N}\left(t_{k}\right)\right)-I_{k}(0)+I_{k}(0)\right\| \\
& \leq 2 N M^{2}\left(\sum_{k=1}^{N} M_{k} E\left\|x^{N}\left(t_{k}\right)\right\|^{2}+\sum_{k=1}^{N}\left\|I_{k}(0)\right\|^{2}\right),
\end{aligned}
$$




$$
\begin{gathered}
E\left\|\int_{0}^{t^{N}} S\left(t^{N}-s\right) g\left(s, x_{s}\right) d W(s)\right\|^{2} \leq \operatorname{tr}(Q) M^{2} \int_{0}^{t^{N}} E\left\|g\left(s, x_{s}\right)\right\|^{2} d s \\
\leq \operatorname{tr}(Q) M^{2} \bar{M}_{g}^{2} \int_{0}^{t^{N}} E\left(\|\varphi\|^{2}+1\right) d s \\
E\left\|\int_{0}^{t^{N}} C\left(t^{N}-s\right) D\left(x_{s}\right) d s\right\|^{2} \leq T M^{2} \bar{M}_{D}^{2} \int_{0}^{t^{N}} E\left(\|\varphi\|^{2}+1\right) d s \\
E\left\|\int_{0}^{t^{N}} S\left(t^{N}-s\right) f\left(s, x_{s}\right) d s\right\|^{2} \leq T M^{2} \bar{M}_{f}^{2} \int_{0}^{t^{N}} E\left(\|\varphi\|^{2}+1\right) d s \\
E\left\|\int_{0}^{t^{N}} S\left(t^{N}-s\right) B u(s) d s\right\|^{2} \\
\leq 8 M_{2} M^{2}\left(\|\xi\|^{2}+\|\varphi(0)\|^{2}+y_{0}^{2}\right. \\
+(T+1) \bar{M}_{D}^{2} \int_{0}^{t^{N}} E\left(\|\varphi\|^{2}+1\right) d s \\
+T \bar{M}_{f}^{2} \int_{0}^{t^{N}} E\left(\|\varphi\|^{2}+1\right) d s \\
+\bar{M}_{g}^{2} \int_{0}^{t^{N}} E\left(\|\varphi\|^{2}+1\right) d s \\
+2 N \sum_{k=1}^{N} N_{k} E\left\|x^{N}\left(t_{k}\right)\right\|^{2}
\end{gathered}
$$

which gives

$$
\begin{aligned}
N \leq & E\left\|\left(\pi x^{N}\right)\left(t^{N}\right)\right\|^{2} \leq 8 E\left\|C\left(t^{N}\right)[\varphi(0)]\right\|^{2}+8 E\left\|S\left(t^{N}\right)\left[y_{0}-D(0, \varphi)\right]\right\|^{2} \\
& +8 E\left\|\int_{0}^{t^{N}} C\left(t^{N}-s\right) D(s, \varphi) d s\right\|^{2}+8 E\left\|\int_{0}^{t^{N}} S\left(t^{N}-s\right) f(s, \varphi) d s\right\|^{2} \\
& +8 E\left\|\int_{0}^{t^{N}} S\left(t^{N}-s\right) g(s, \varphi) d W(s)\right\|^{2}+8 E\left\|\sum_{0<t_{k}<t^{N}} C\left(t^{N}-t_{k}\right) I_{k}\left(x^{N}\left(t_{k}\right)\right)\right\|^{2} \\
& +8 E\left\|\sum_{0<t_{k}<t^{N}} S\left(t^{N}-t_{k}\right) \tilde{I}_{k}\left(x^{N}\left(t_{k}\right)\right)\right\|^{2}+8 E\left\|\int_{0}^{t^{N}} S\left(t^{N}-s\right) B u(s) d s\right\|^{2}
\end{aligned}
$$


Mathematical Problems in Engineering

$$
\begin{aligned}
\leq L+8 M^{2}[ & T \bar{M}_{D}^{2} N+T \bar{M}_{f}^{2} N+\operatorname{tr}(Q) \bar{M}_{g}^{2} N+2 N M^{2} \sum_{k=1}^{n} M_{k}+2 N M^{2} \sum_{k=1}^{n} N_{k} \\
& \left.+8 M_{2}\left(T \bar{M}_{D}^{2} N+T \bar{M}_{f}^{2} N+\operatorname{tr}(Q) \bar{M}_{g}^{2} N+2 N M^{2} \sum_{k=1}^{n} M_{k}+2 N M^{2} \sum_{k=1}^{n} N_{k}\right)\right]
\end{aligned}
$$

where

$$
\begin{aligned}
L=8 M^{2}\left[E\left\|x_{0}\right\|^{2}+E\left\|y_{0}\right\|^{2}+T \bar{M}_{D}^{2}+T \bar{M}_{f}^{2}+\operatorname{tr}(Q) \bar{M}_{g}^{2}+2 M^{2} \sum_{k=1}^{n} M_{k}+2 M^{2} \sum_{k=1}^{n} N_{k}\right. \\
\left.+8 M_{2}\left(T \bar{M}_{D}^{2}+T \bar{M}_{f}^{2}+\operatorname{tr}(Q) \bar{M}_{g}^{2}+2 M^{2} \sum_{k=1}^{n} M_{k}+2 M^{2} \sum_{k=1}^{n} N_{k}\right)\right]
\end{aligned}
$$

Dividing both sides of (3.16) by $N$ and taking limit as $N \rightarrow \infty$, we obtain that

$$
\begin{aligned}
& 8 M^{2}\left[T \bar{M}_{D}^{2}+T \bar{M}_{f}^{2}+\operatorname{tr}(Q) \bar{M}_{g}^{2}+2 M^{2} \sum_{k=1}^{n} M_{k}+2 M^{2} \sum_{k=1}^{n} N_{k}\right. \\
& \left.\quad+8 M_{2}\left(T \bar{M}_{D}^{2}+T \bar{M}_{f}^{2}+\operatorname{tr}(Q) \bar{M}_{g}^{2}+2 M^{2} \sum_{k=1}^{n} M_{k}+2 M^{2} \sum_{k=1}^{n} N_{k}\right)\right] \geq 1
\end{aligned}
$$

which is a contradiction by (3.7). Thus, $\pi\left(B_{N}\right) \subset B_{N}$, for some positive number $N$.

In what follows, we aim to show that the operator $\pi$ has a fixed point on $B_{N}$, which implies that (1.1) is controllable. To this end, we decompose $\pi$ as follows:

$$
\pi=\pi_{1}+\pi_{2}
$$

where $\pi_{1}, \pi_{2}$ are defined on $B_{N}$, respectively, by

$$
\begin{aligned}
&\left(\pi_{1} x\right)(t)= S(t)\left[y_{0}-D(0, \varphi)\right]+\int_{0}^{t} C(t-s) D(0, \varphi) d s+\int_{0}^{t} S(t-s) f\left(s, x_{s}\right) d s \\
&+\sum_{0<t_{k}<t} C\left(t-t_{k}\right) I_{k}\left(x\left(t_{k}\right)\right)+\sum_{0<t_{k}<t} S\left(t-t_{k}\right) \tilde{I}_{k}\left(x\left(t_{k}\right)\right), \\
&\left(\pi_{2} x\right)(t)=C(t) \phi(0)+\int_{0}^{t} S(t-s) g\left(s, x_{s}\right) d W(s)+\int_{0}^{t} S(t-s) B u(s) d s .
\end{aligned}
$$

Lemma 3.4. The operator $\pi_{1}$ as above is contractive. 
Proof. Let $x, y \in B_{N}$. It follows from assumptions (A1)-(A4) and Hölder's inequality that

$$
\begin{aligned}
E\left\|\left(\pi_{1} x\right)(t)-\left(\pi_{1} y\right)(t)\right\|^{2} \\
\leq 5 E\|S(t)[D(0, \varphi)-D(0, \phi)]\|^{2} \\
\quad+5 E\left\|\int_{0}^{t} C(t-s)[D(0, \varphi)-D(0, \phi)] d s\right\|^{2}+5 E\left\|\int_{0}^{t} S(t-s)[f(s, \varphi)-f(s, \phi)] d s\right\|^{2} \\
\quad+5 E\left\|\sum_{0<t_{k}<t} C\left(t-t_{k}\right)\left[I_{k}\left(x\left(t_{k}\right)\right)-I_{k}\left(y\left(t_{k}\right)\right)\right]\right\|^{2} \\
\quad+5 E\left\|\sum_{0<t_{k}<t} S\left(t-t_{k}\right)\left[\tilde{I}_{k}\left(x\left(t_{k}\right)\right)-\tilde{I}_{k}\left(y\left(t_{k}\right)\right)\right]\right\|^{2} \\
\leq 5 M^{2} M_{D}^{2} \sup _{s \in[0, T]} E\|x(s)-y(s)\|^{2}+5 T M^{2} M_{D}^{2} \sup _{s \in[0, T]} E\|x(s)-y(s)\|^{2} \\
\quad+5 T M^{2} M_{f}^{2} \sup _{s \in[0, T]} E\|x(s)-y(s)\|^{2}+5 n M^{2} \sum_{0<t_{k}<t} M_{k} E\left\|x\left(t_{k}\right)-y\left(t_{k}\right)\right\|^{2} \\
\quad+5 n M^{2} \sum_{0<t_{k}<t} N_{k} E\left\|x\left(t_{k}\right)-y\left(t_{k}\right)\right\|^{2}
\end{aligned}
$$

which deduces

$$
\begin{aligned}
\sup _{s \in[0, T]} & E\left\|\left(\pi_{1} x\right)(s)-\left(\pi_{1} y\right)(s)\right\|^{2} \\
& \leq 5 M^{2}\left[M_{D}^{2}+T M_{D}^{2}+T M_{f}^{2}+n \sum_{i=0}^{n} M_{k}+n \sum_{i=0}^{n} N_{k}\right] \sup _{s \in[0, T]} E\|x(s)-y(s)\|^{2}
\end{aligned}
$$

and the lemma follows.

Lemma 3.5. The operator $\pi_{2}$ is compact.

Proof. Let $N>0$ be such that $\pi_{2}\left(B_{N}\right) \subset B_{N}$.

We first need to prove that the set of functions $\pi_{2}\left(B_{N}\right)$ is equicontinuous on $[0, T]$. Let $0<\varepsilon<t<T$ and $\delta>0$ such that $\left\|S(s) x-S\left(s^{\prime}\right) x\right\|^{2}<\varepsilon$ and $\left\|C(s) x-C\left(s^{\prime}\right) x\right\|^{2}<\varepsilon$, for every $s, s^{\prime} \in[0, T]$ with $\left|s-s^{\prime}\right| \leq \delta$. For $x \in B_{N}$ and $0<|h|<\delta$ with $t+h \in[0, T]$ we have

$$
\begin{aligned}
& E\left\|\left(\pi_{2} x\right)(t+h)-\left(\pi_{2} x\right)(t)\right\|^{2} \\
& \leq 3 E\|[C(t+h)-C(t)] \phi(0)\|^{2} \\
& \quad+3 E\left\|\int_{0}^{t}[S(t+h-s)-S(t-s)] g\left(s, x_{s}\right) d W(s)-\int_{t}^{t+h} S(t+h-s) g\left(s, x_{s}\right) d W(s)\right\|^{2} \\
& \quad+3 E\left\|\int_{0}^{t}[S(t+h-s)-S(t-s)] B u(s) d s-\int_{t}^{t+h} S(t+h-s) B u(s) d s\right\|^{2}
\end{aligned}
$$


Mathematical Problems in Engineering

$$
\begin{aligned}
\leq & 3 \varepsilon E\|\phi(0)\|^{2}+6 \operatorname{tr}(Q) M^{2} \int_{t}^{t+h} E\left\|g\left(s, x^{\prime}(s), x_{s}\right)\right\|^{2} d s+6 M^{2} \int_{t}^{t+h} E\|B u(s)\|^{2} d s \\
& +6 M^{2} \int_{0}^{t} E\|B u(s)\|^{2} d s+6 \operatorname{tr}(Q) \int_{0}^{t} E\left\|[S(t+h-s)-S(t-s)] g\left(s, x_{s}\right)\right\|^{2} d s \\
\leq & 4 \varepsilon E\left\|x_{0}\right\|^{2}+4 \varepsilon E\|g(x)\|^{2}+4 \varepsilon \operatorname{tr}(Q) \int_{0}^{t} E\left\|g\left(s, x_{s}\right)\right\|^{2} d s \\
& +4 \operatorname{tr}(Q) M^{2} \int_{t}^{t+h} E\left\|g\left(s, s^{x}(s)\right)\right\|^{2} d s .
\end{aligned}
$$

Noting that $E\left\|g\left(s, s^{x}(s)\right)\right\|^{2} \leq h_{N}(s) \in L^{1}([0, T])$, we see that $\pi_{2}\left(B_{N}\right)$ is equicontinuous on [0, $T]$.

We next need to prove that $\pi_{2}$ maps $B_{N}$ into a precompact set in $B_{N}$. That is, for every fixed $t \in[0, T]$, the set $V(t)=\left\{\left(\pi_{2} x\right)(t): x \in B_{N}\right\}$ is precompact in $B_{N}$. It is obvious that $V(0)=\left\{\left(\pi_{2} x\right)(0)\right\}$ is precompact. Let $0<t \leq T$ be fixed and $0<\varepsilon<t$. For $x \in B_{N}$, define

$$
\begin{aligned}
\left(\pi_{2}^{\varepsilon} x\right)(t) & =C(t) \phi(0)+\int_{0}^{t-\varepsilon} S(t-s) g\left(s, x_{s}\right) d W(s)+\int_{0}^{t-\varepsilon} S(t-s) B u(s) d s \\
& =C(t) \phi(0)+S(\varepsilon) \int_{0}^{t-\varepsilon} S(t-\varepsilon-s) g\left(s, x_{s}\right) d W(s)+S(\varepsilon) \int_{0}^{t-\varepsilon} S(t-\varepsilon-s) B u(s) d s .
\end{aligned}
$$

Since $C(t), S(t), t>0$, are compact, it follows that $V_{\varepsilon}(t)=\left\{\left(\pi_{2}^{\varepsilon} x\right)(t): x \in B_{N}\right\}$ is precompact in $H$ for every $0<\varepsilon<t$. Moreover, for each $x \in B_{N}$, we have

$$
\begin{aligned}
E\left\|\left(\pi_{2} x\right)(t)-\left(\pi_{2}^{\varepsilon} x\right)(t)\right\|^{2} & \leq 2 \operatorname{tr}(Q) M^{2} \int_{t-\varepsilon}^{t} E\left\|g\left(s, x_{s}\right)\right\|^{2} d s+2 M^{2} \int_{t-\varepsilon}^{t} E\|B u(s)\|^{2} d s \\
& \leq \varepsilon 2 M^{2}\left[\operatorname{tr}(Q) E\left(\|\varphi\|^{2}+1\right)+U\right] \longrightarrow 0 \text { as } \varepsilon \longrightarrow 0^{+}
\end{aligned}
$$

which means that there are precompact sets arbitrary close to the set $V(t)$. Thus, $V(t)$ is precompact in $B_{N}$.

Finally, from the assumptions on $g$, it is obvious that $\pi_{2}$ is continuous. Thus, ArzeláAscoli theorem yields that $\pi_{2}$ is compact. Therefore, $\pi$ is a condensing map on $B_{N}$.

\section{Applications}

In this section, we now give an example to illustrate the theory obtained. Considering the following impulsive neutral second-order stochastic differential equation:

$$
\begin{gathered}
d\left[\frac{\partial x(t, z)}{\partial t}+a(t) x(t, z)\right]=\frac{\partial^{2}}{\partial z^{2}} x(t, z) d t+\sigma(t, x(t, z)) d W(t), \quad t \in[0,1] \\
x(t, 0)=x(t, \pi)=0, \quad t \in[0,1], \quad \frac{\partial x(0, z)}{\partial t}=x_{1}(z), \quad z \in[0, \pi] \\
\Delta x\left(t_{k}\right)(z)=I_{k}\left(x\left(t_{k}\right)\right)(z), \quad \Delta x^{\prime}\left(t_{k}\right)(z)=\tilde{I}_{k}\left(x\left(t_{k}\right)\right)(z), \quad t=t_{k}
\end{gathered}
$$


to rewrite (4.1) into the abstract form of (1.1), let $H=L^{2}[0, \pi], A: H \rightarrow H$ be an operator by $A x=x^{\prime \prime}$ with domain

$$
D(A)=\left\{x \in H: x, x^{\prime} \text { are absolutely continuous, } x^{\prime \prime} \in H, x(0)=x(\pi)=0\right\} .
$$

It is well known that $A$ is the infinitesimal generator of a strongly continuous cosine family $\{C(t): t \in R\}$ in $H$ and is given by

$$
C(t) x=\sum_{n=1}^{\infty} \cos (n t)\left\langle x, e_{n}\right\rangle e_{n}, \quad x \in H,
$$

where $e_{n}(\xi)=\sqrt{2 / \pi} \sin (n \xi)$ and $i=1,2, \ldots$ is the orthogonal set of eigenvalues of $A$. The associated sine family $\{S(t): t>0\}$ is compact and is given by

$$
S(t) x=\sum_{n=1}^{\infty} \frac{1}{n} \sin (n t)\left\langle x, e_{n}\right\rangle e_{n}, \quad x \in H .
$$

Thus, we can impose some suitable conditions on the above functions to verify the condition in Theorem 3.2.

\section{Conclusions}

In this paper, we have studied the controllability of second-order impulsive evolution equations. Through the Sadovskii fixed point theorem and the theory of strongly continuous cosine families of operators, we have investigated the sufficient conditions for the controllability of the system considered. At last, an example is provided to show the usefulness and effectiveness of proposed controllability results.

\section{Acknowledgments}

This work was supported in part by the Key Project of the National Nature Science Foundation of China (no. 61134009), the National Nature Science Foundation of China (no. 60975059), Specialized Research Fund for the Doctoral Program of Higher Education from Ministry of Education of China (no. 20090075110002), Specialized Research Fund for Shanghai Leading Talents, and Project of the Shanghai Committee of Science and Technology (nos. 11XD1400100, 11JC1400200, 10JC1400200).

\section{References}

[1] Y.-K. Chang, "Controllability of impulsive functional differential systems with infinite delay in Banach spaces," Chaos, Solitons and Fractals, vol. 33, no. 5, pp. 1601-1609, 2007.

[2] R. Sakthivel, "Approximate controllability of impulsive stochastic evolution equations," Funkcialaj Ekvacioj, vol. 52, no. 3, pp. 381-393, 2009.

[3] Y. Ren, L. Hu, and R. Sakthivel, "Controllability of impulsive neutral stochastic functional differential inclusions with infinite delay," Journal of Computational and Applied Mathematics, vol. 235, no. 8, pp. 2603-2614, 2011. 
[4] S. K. Ntouyas and D. O'Regan, "Some remarks on controllability of evolution equations in Banach spaces," Electronic Journal of Differential Equations, vol. 2009, pp. 1-6, 2009.

[5] J. Y. Park, S. H. Park, and Y. H. Kang, "Controllability of second-order impulsive neutral functional differential inclusions in Banach spaces," Mathematical Methods in the Applied Sciences, vol. 33, no. 3, pp. 249-262, 2010.

[6] R. Sakthivel, N. I. Mahmudov, and J. H. Kim, "On controllability of second order nonlinear impulsive differential systems," Nonlinear Analysis, vol. 71, no. 1-2, pp. 45-52, 2009.

[7] M. A. Shubov, C. F. Martin, J. P. Dauer, and B. P. Belinskiy, "Exact controllability of the damped wave equation," SIAM Journal on Control and Optimization, vol. 35, no. 5, pp. 1773-1789, 1997.

[8] J. Luo, "Fixed points and exponential stability of mild solutions of stochastic partial differential equations with delays," Journal of Mathematical Analysis and Applications, vol. 342, no. 2, pp. 753-760, 2008.

[9] J. Luo and T. Taniguchi, "Fixed points and stability of stochastic neutral partial differential equations with infinite delays," Stochastic Analysis and Applications, vol. 27, no. 6, pp. 1163-1173, 2009.

[10] T. A. Burton, "Stability by fixed point theory or Liapunov theory: a comparison," Fixed Point Theory, vol. 4, no. 1, pp. 15-32, 2003.

[11] T. A. Burton, "Fixed points, stability, and exact linearization," Nonlinear Analysis, vol. 61, no. 5, pp. 857-870, 2005.

[12] T. A. Burton and T. Furumochi, "Asymptotic behavior of solutions of functional differential equations by fixed point theorems," Dynamic Systems and Applications, vol. 11, no. 4, pp. 499-519, 2002.

[13] T. A. Burton and B. Zhang, "Fixed points and stability of an integral equation: nonuniqueness," Applied Mathematics Letters, vol. 17, no. 7, pp. 839-846, 2004.

[14] K. Sobczyk, Stochastic Differential Equations, vol. 40 of Mathematics and Its Applications (East European Series), Kluwer Academic, Dordrecht, The Netherlands, 1991.

[15] W. E. Fitzgibbon, "Global existence and boundedness of solutions to the extensible beam equation," SIAM Journal on Mathematical Analysis, vol. 13, no. 5, pp. 739-745, 1982.

[16] M. Benchohra, J. Henderson, and S. K. Ntouyas, "Existence results for impulsive semilinear neutral functional differential equations in Banach spaces," Memoirs on Differential Equations and Mathematical Physics, vol. 25, pp. 105-120, 2002.

[17] X. Liu and A. R. Willms, "Impulsive controllability of linear dynamical systems with applications to maneuvers of spacecraft," Mathematical Problems in Engineering, vol. 2, no. 4, pp. 277-299, 1996.

[18] E. Hernández, M. Pierri, and G. Goncalves, "Existence results for an impulsive abstract partial differential equation with state-dependent delay," Computers $\mathcal{E}$ Mathematics with Applications, vol. 52, no. 3-4, pp. 411-420, 2006.

[19] G. Da Prato and J. Zabczyk, Stochastic Equations in Infinite Dimensions, vol. 44 of Encyclopedia of Mathematics and Its Applications, Cambridge University Press, Cambridge, Mass, USA, 1992.

[20] H. O. Fattorini, Second Order Linear Differential Equations in Banach Spaces, vol. 108 of North-Holland Mathematics Studies, North-Holland, Amsterdam, The Netherlands, 1985.

[21] C. C. Travis and G. F. Webb, "Cosine families and abstract nonlinear second order differential equations," Acta Mathematica Academiae Scientiarum Hungaricae, vol. 32, no. 1-2, pp. 75-96, 1978.

[22] B. N. Sadovskiı̌, “On a fixed point principle," Functional Analysis and Its Applications, vol. 1, no. 2, pp. 74-76, 1967. 


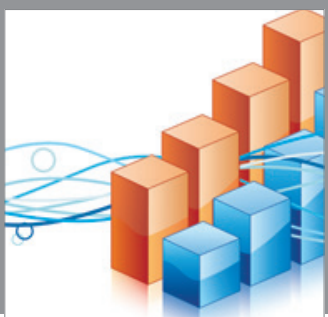

Advances in

Operations Research

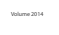

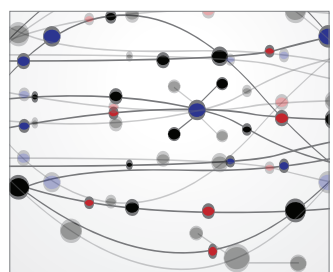

\section{The Scientific} World Journal
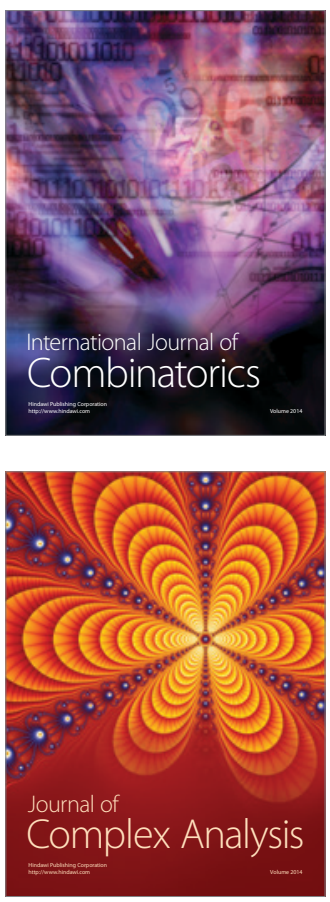

International Journal of

Mathematics and

Mathematical

Sciences
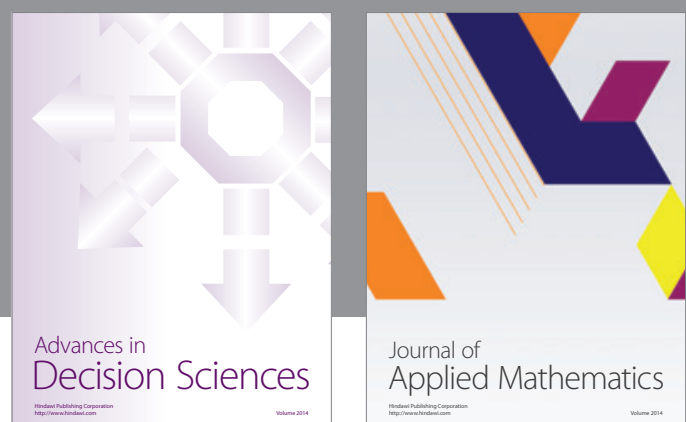

Journal of

Applied Mathematics
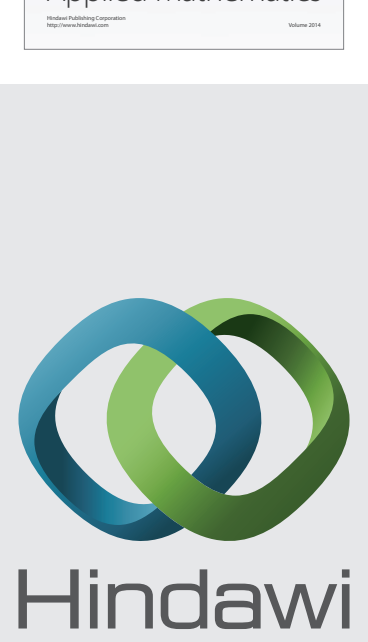

Submit your manuscripts at http://www.hindawi.com
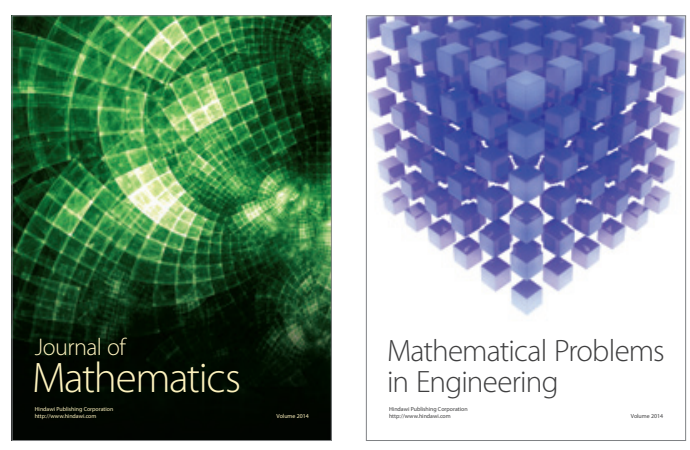

Mathematical Problems in Engineering
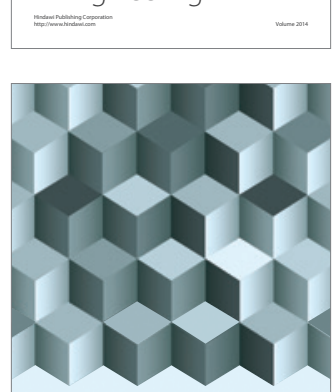

Journal of

Function Spaces
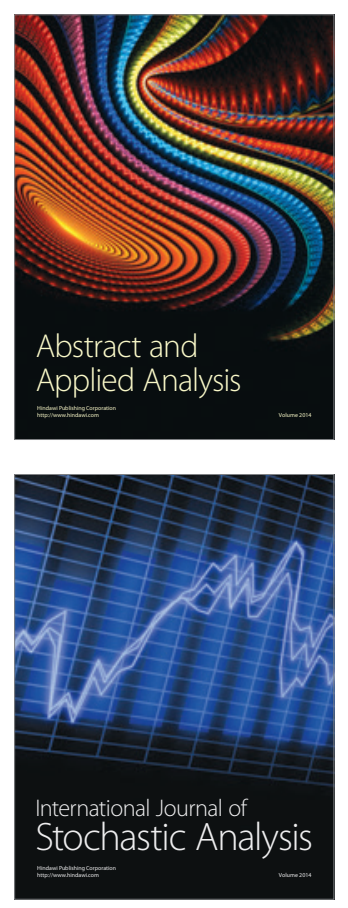

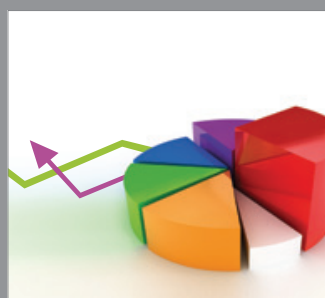

ournal of

Probability and Statistics

Promensencen
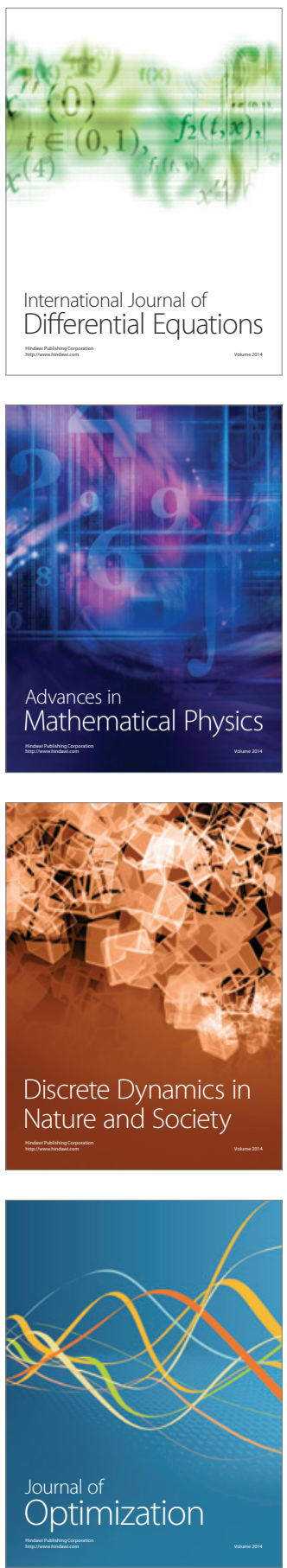